\title{
A High Field Magnet Design for A Future Hadron Collider*
}

\author{
R. Gupta, K. Chow, D. Dietderich, S. Gourlay, G. Millos, A. McInturff, R. Scanlan \\ Lawrence Berkeley National Laboratory, Berkeley, CA 94720, USA
}

\section{S. Ramberger, S. Russenschuck \\ CERN, Geneva, Switzerland}

\begin{abstract}
US high energy physics community is exploring the possibilities of building a Very Large Hadron Collider (VLHC) after the completion of LHC. This paper presents a high field magnet design option based on $\mathrm{Nb}_{3} \mathrm{Sn}$ technology. A preliminary magnetic and mechanical design of a 14-16 T, 2-in-1 dipole based on the "common coil design" approach is presented. The computer code ROXIE has been upgraded to perform the field quality optimization of magnets based on the racetrack coil geometry. A magnet R\&D program to investigate the issues related to high field magnet designs is also outlined.
\end{abstract}

\section{INTRODUCTION}

As a first step towards a new high field magnet program at the Lawrence Berkeley National Laboratory (LBNL), a moderate field, 2-in-1 dipole magnet, based on the common coil design [1] has been built $[2,3]$. This magnet uses the brittle $\mathrm{Nb}_{3} \mathrm{Sn}$ conductor. It will, however, generate only $\sim 7$ tesla field since an existing lower critical current density conductor was used for expediency. This moderate field magnet is being used to test the basic design and to carry out studies relevant to the next high field magnet. This paper presents an initial design of the high field magnet with a higher performance $\mathrm{Nb}_{3} \mathrm{Sn}$ conductor.

The basic geometry and the philosophy of the common coil design has been described elsewhere [1]. In this design the main coils are shared between the two apertures in a block geometry that is suitable for high field magnets. The sharing of some coils between the two apertures was proposed earlier in a "Double Dipole" design [4] also. In the common coil design none of the conductor has to cross the vertical axis. This allows a larger bend radius for the conductors in a complete 2-d "conductor-friendly" geometry that is suitable for brittle materials. The design also allows a modular and flexible R\&D program to carry out systematic investigations of issues related to the high field magnet technology. These include (a) changing the pre-stress, (b) replacing or adding coils perhaps with a different cable type and/or geometry, (c) changing components of the mechanical support structure, $(d)$ changing the magnet aperture (after the coils are built) to increase the bore field from $14 \mathrm{~T}$ to $16 \mathrm{~T}$ and (e) adopting the design to produce lower cost magnets.

The major difference between the moderate field and the high field magnet design is the way horizontal component of the Lorentz forces is handled. In the moderate field magnet, the coils would continue to have a pre-stress to the design

Manuscript received September 15, 1998.

${ }^{*}$ This work is supported by the U.S. Department of Energy under contract No. DE-AD03-76SF00098. field. In the high magnet the coil will have a small pre-stress only at low field and the inner surface will be free at high field. At low fields a low initial pre-stress will assure contact to the support structure. At high fields the Lorentz forces would continue to maintain that contact. The support structure is being designed to withstand the large accumulated Lorentz forces at 16 tesla.

\section{MAGNETIC DESIGN}

The primary purpose of this magnet is to demonstrate the viability of the dipoles based on the common coil design in a mechanical structure that is suitable for high field accelerator magnets. The field quality issues will be addressed later; first through the computer modeling and then by adding the spacers and auxiliary coils in future magnets.

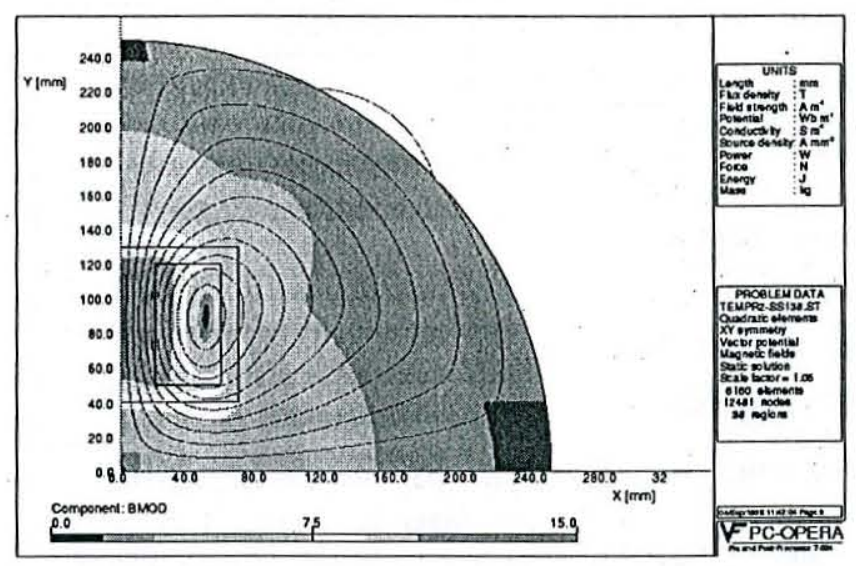

Fig. 1. An OPERA-2d model of the present design of $\sim 14$ T, 2-in-1 magnet. The model shows the field lines and contour in a quadrant of the coldmass.

An OPERA-2d model of the present design is shown in Fig. 1 and the major parameters are given in Table 1. This design uses three layers of coils (a total of six coils in two apertures). The computed short sample (quench field) in magnet bore is 13.8 tesla in the $40 \mathrm{~mm}$ aperture mode and $16.2 \mathrm{~T}$ in the $10 \mathrm{~mm}$ aperture mode. These calculations do not include the cable degradation due to stresses. The outer two layers will be in a 'double pancake' coil geometry and the inner in a single pancake.

A new scheme is adopted here for grading the conductor for better efficiency and more flexibility. The primary purpose of grading is to put lower current density in the cables in the inner layer(s) where the field is higher and higher current density in the outer layer(s) where the field is lower. In a conventional design it is obtained by changing the cable thickness by changing the wire diameter. This however, in- 
creases the relative fraction of the cable insulation in the coil. In the proposed design the same cable thickness and thus the similar fraction of cable insulation is used in each layer. The grading is achieved by changing the cable width by changing the number of wires (strands) in it. Since the wire diameter is not changed a greater flexibility in design is possible - the relative grading between layers can be changed by changing the cable width (by changing the number of wires) even after the superconducting wire is purchased. The present design has 40 strands in the inner layer and 26 in the outer two.

TABLE I

THE MAJOR PARAMETERS OF THE PRESENT DESIGN FOR A 40 MM APERTURE COMMON COIL DESIGN MAGNET (IN SECOND COLUMN THE PARAMETERS FOR A 10 MM APERTURE CONFIGURATION ARE GIVEN IN PARENTHESIS).

\begin{tabular}{|l|l|}
\hline Coil aperture (mm) & $40(10)$ \\
\hline Number of layers & 3 \\
\hline Computed quench field at $4.2 \mathrm{~K}(\mathrm{~T})$ & $13.8(16.2)$ \\
\hline Peak Fields, inner \& outer layers (T) & 15.0 \& $10.5(16.3 \& 11.4)$ \\
\hline Quench current, inner \& outer layers (kA) & 12.0 \& $12.0(8.7 \& 13.0)$ \\
\hline Wire Non-Cu J sc $44.2 \mathrm{~K}, 12 \mathrm{~T}\}\left(\mathrm{A} / \mathrm{mm}^{2}\right)$ & 2000 \\
\hline Strand diameter (mm) & 0.8 \\
\hline No. of strands, inner \& outer layers & 40,26 \\
\hline Cable width, inner \& outer layer (mm) & $16.5,10.7$ \\
\hline Cu/Non-Cu ratio, inner \& outer & $0.7,1.7$ \\
\hline No. of turns (total) & 120 \\
\hline Height of each layer (mm) & 70 \\
\hline Bore spacing (mm) & 170 \\
\hline Minimum coil bend radius (mm) & 50 \\
\hline Yoke outer radius (mm) & 250 \\
\hline
\end{tabular}

The center-to-center spacing between the two apertures is $170 \mathrm{~mm}$ and height of each layer is $70 \mathrm{~mm}$. This gives a minimum coil bend radius (in the ends) of $50 \mathrm{~mm}$, which is about an order of magnitude more than that in conventional block designs or in $\operatorname{cosine}(\theta)$ designs. The width of the iron insert between the two apertures is $80 \mathrm{~mm}$. The iron insert reduces the coupling (cross-talk) between the two apertures and gives a higher short sample field. The iron insert, however, generates large saturation induced harmonics in this geometry. The issue of iron insert and the optimization of saturation induced harmonics will be examined later.

The yoke outer radius is only $250 \mathrm{~mm}$. The compact nature of the design is related to the magnet geometry. When this design is adopted for a $50 \mathrm{~mm}$ aperture magnet and compared with D20 (a similar field and same aperture dipole built and tested earlier at LBNL [5]), the yoke or coldmass area per aperture in this design is only a quarter of that in D20.

\section{MAGNETIC DESIGN OPTIMIZATION WITH ROXIE}

The computer code ROXIE $[6,7]$ will be used in optimizing the field quality and magnetic design of an accelerator magnet built on this concept. This program has been used in designing a number of LHC and other magnets based on a $\operatorname{cosine}(\theta)$ coil geometry. ROXIE has now been upgraded to optimize a magnet built with racetrack coils inside an arbitrarily shaped iron aperture. ROXIE can also minimize the end harmonics in such a geometry.

The primary parameters in coil cross-section optimization are (a) internal spacers within each coil layer (similar to the wedges in $\operatorname{cosine} \theta$ magnets) and (b) coil height and beginning position of each layer (similar to the pole angle). The coil geometry must have an up-down asymmetry with respect to the midplane in each aperture to compensate for the coupling between the two apertures. The shape and dimensions of various end spacers and length of coil are used as parameters to minimize both normal and skew end harmonics. The iron saturation is minimized by shaping the iron inner surface between the two apertures and around the coil modules. In addition, the holes and cutouts (in the iron cross section) and the yoke outer surface can be used as parameters in minimizing saturation induced normal and skew harmonics. A computer model of the present design (which is not a freld quality design) is shown in Fig. 2.

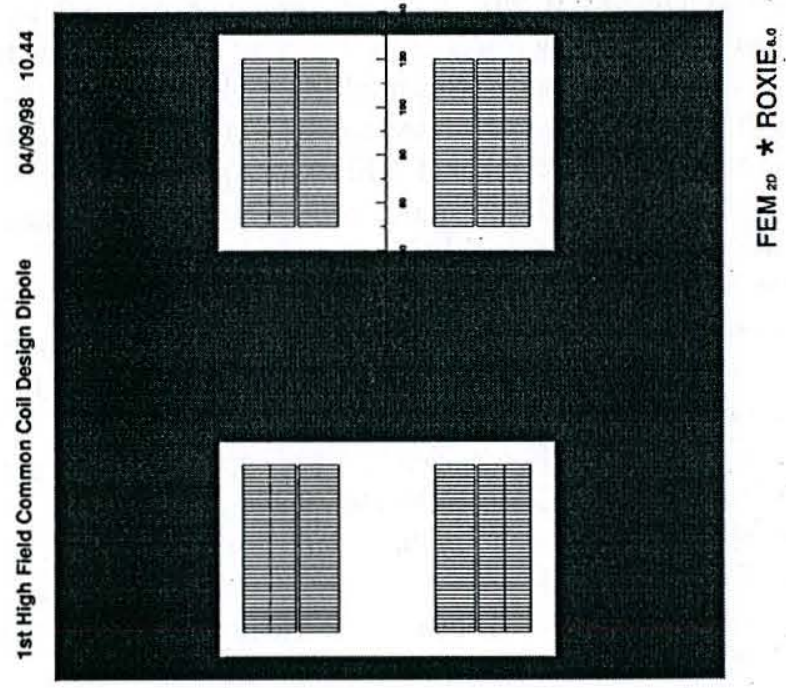

Fig. 2. A ROXIE model of the high field common coil design magnet. The figure shows only a part of the iron and 3-layer of coils with iron inserts between the two apertures.

\section{MECHANICAL DESIGN}

At fields of 14 to 16 Tesla the mechanical design is driven by two requirements: supporting high Lorentz forces and managing coil stresses. The integrated Lorentz forces act to push the magnet coils apart; a rigid support structure design is required to minimize coil displacements and maintain magnet integrity. Within the coil winding the Lorentz forces act to compress the winding (transverse to winding direction); a stiff and strong coil design is required to minimize coil deformation and withstand internal stresses. In addition to the structural concerns, thermal conductance is an issue because adequate heat transfer is required for thermal stabil- 
ity. Lastly, the mechanical design is based on criteria applicable to this R\&D program: flexibility, modularity, short turnaround time, and ease of fabrication.

The current mechanical design is a "dedicated component" design. The magnet is separated into three main components and each serves a dedicated function. The magnet core resides within the iron yoke and serves to package the parts within the core (coils, islands, end shoes, spacers, etc.), the iron yoke surrounds the magnet core and serves as a flux return, and an external wire wrap is used around the iron yoke to provide preload and structural support. In contrast to the dedicated component design is an "integrated component" design wherein each component serves multiple functions (e.g. the function of structural support is shared between magnet core, iron yoke, and wire wrap). The dedicated component design provides the flexibility and modularity appropriate for R\&D magnets; since the magnet core does not in:corporate any support function, different magnet core designs can be used with the same external support (provided the support is adequately strong and rigid). Such a system lends itself to a program of systematic investigations. On the other hand, an integrated component design is inherently more efficient and is suited for production magnet designs.

\section{A. External Support Design}

Figures $3 \mathrm{a}$ and $3 \mathrm{~b}$ show the horizontal and vertical force densities in a coil cross section for one quadrant of the magnet winding (with only main coils). At $14 \mathrm{~T}$ bore field, the total integrated horizontal force is $19 \mathrm{MN}$ per magnet straight section length in meters (137 MPa distributed over the racetrack edge area); total integrated vertical force is $1.6 \mathrm{MN} / \mathrm{m}$ (21 MPa distributed over the racetrack face area). Total integrated forces are the main factor in designing the external magnet support; the structure needs to be strong enough to support the integrated outward forces.

An external wrap of stainless steel wire has been successfully used for applying preload and support in a high field $\operatorname{cosine}(\theta)$ magnet [5]. The current design uses a similar wire wrap technique for support. The wire is wound onto a bobbin placed around the iron yokes and locked into the bobbin after each wrap layer. Shaping the outer yoke perimeter provides control of horizontal and vertical preload. Design of the yoke gaps also determines effective preload and support. The wire wrap has two roles: vertical preload on the coils and support against Lorentz forces.

In the vertical direction the coils are preloaded to a level which prevents the coil inner turn from separating from the island when the magnet is energized. Magnet training is expected to be reduced by preventing energy release due to coil movement. In the horizontal direction the preload is small. The preload ensures contact between the coil outer surface and support structure, but at high fields the inner coil surface is free. The accumulated Lorentz forces act mostly in the horizontal direction. The forces push on the iron yoke, which transfers the load to the external wire wrap support structure.

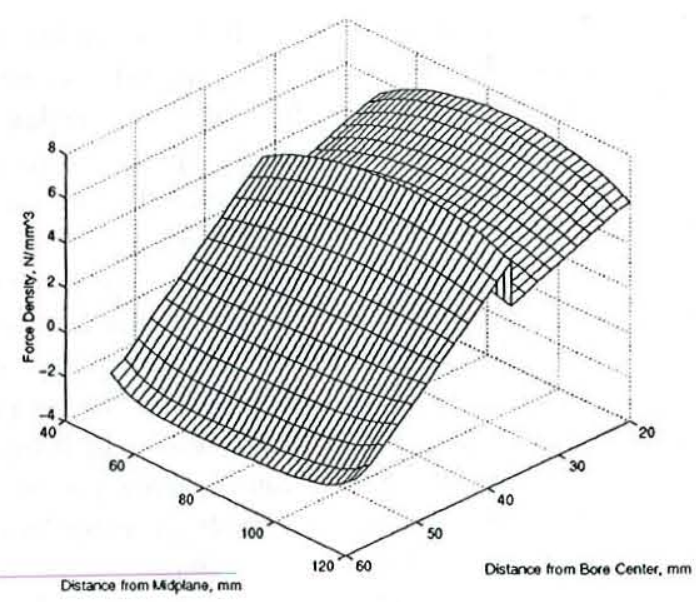

Fig. 3a. Horizontal force density

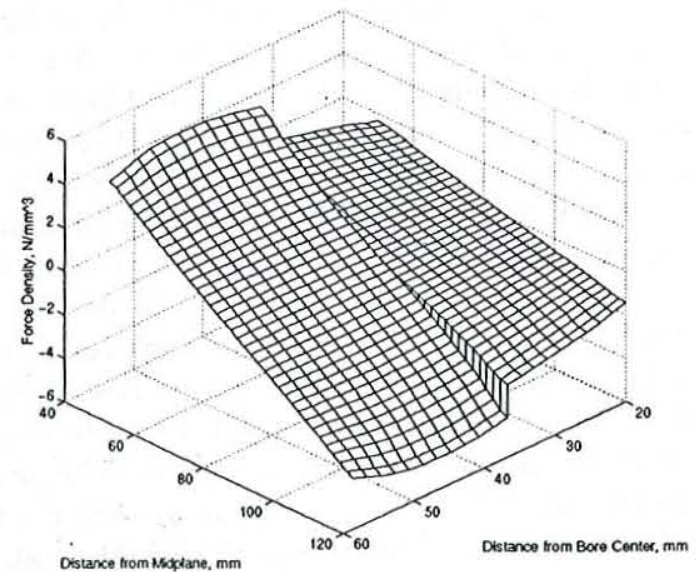

Fig. 3b. Vertical force density

Fig. 3. Horizontal and vertical force densities at $14 \mathrm{~T}$ bore field for coil cross section in quadrant 1 of high field common coil magnet. Positive values denote forces outward from magnet center.

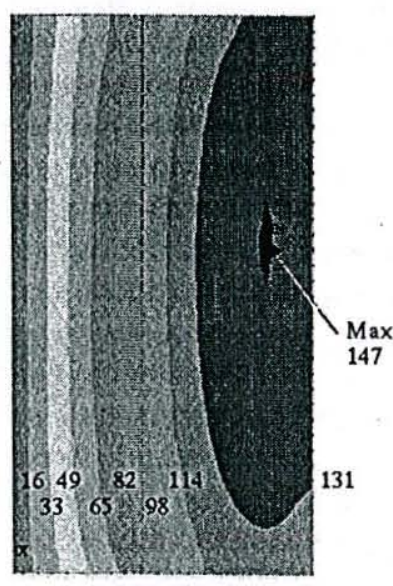

Horizontal Stress

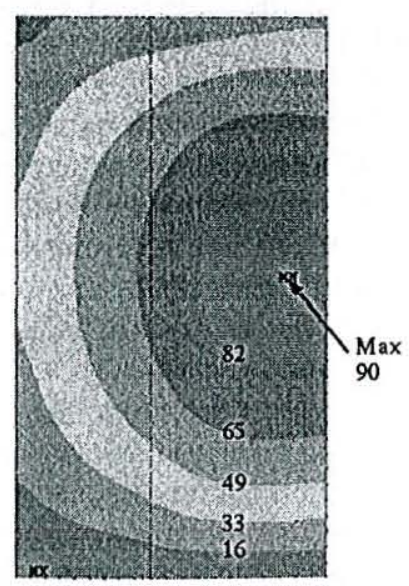

Vertical Stress
Fig. 4. Horizontal and vertical coil stresses for coil cross section in quadrant 1 of high field $(14 \mathrm{~T})$ common coil magnet. All values are compressive in $\mathrm{MPa}$. 


\section{B. Coil Stresses}

The force distribution within a coil cross section is the source of internal coil stresses when the magnet is energized. Note that for the common coil design the forces are low near the cross section center and increase (in opposite directions) towards the sides, effectively compressing the coil in both horizontal and vertical directions (see Fig. 4).

Epoxy impregnation is used to provide support for the brittle superconductors. The extra support provided by the epoxy allows normal handling of the heat treated brittle coils. The epoxy also serves to increase the mean stiffness of the composite coil. Increasing the stiffness results in lower deformation of the coil winding under Lorentz forces. The elastic modulus of epoxy impregnated $\mathrm{Nb}_{3} \mathrm{Sn}$ cable in compression has been measured at up to $53 \mathrm{GPa}$ transverse to the winding direction [8].

If no structural elements are incorporated into the coil winding, the stresses accumulate to very high levels (up to $150 \mathrm{MPa}$ for this high field common coil design). A 16 Tesla dipole magnet is currently under development at Texas A\&M University which uses a sophisticated rib and plate structure to provide structural support within the coil cross section [9]. The common coil design is amenable to similar stress management schemes and they may be incorporated later, when and if necessary. However, previous test results of an epoxy impregnated $\mathrm{Nb}_{3} \mathrm{Sn}$ dipole indicate that the high coil stresses do not significantly degrade conductor performance at up to 13.5 Tesla with comparable stress levels [5]. For the current common coil design, no structural support is incorporated into the coil winding. Moreover, it may be pointed out that although the stress accumulates to high levels in the coil, the high stresses occur at low field locations (see Fig. 1 and Fig. 4).

\section{MAGNET R\&D PROGRAM}

The design approach described here allows a systematic and efficient magnet R\&D program. One major design and performance issue in very high field $\mathrm{Nb}_{3} \mathrm{Sn}$ magnets is the amount of cable degradation due to large stress accumulation. The "in-magnet" situation is better since the place of the highest stress accumulation is also the place of the highest field margin. Moreover, the tests done in small cable samples to determine the degradation do not always simulate the actual magnet conditions. This design allows simulating "actual magnet test situation" by creating a field of 16.2 tesla (computed short sample assuming no stress degradation) in the $10 \mathrm{~mm}$ aperture mode (see Table 1) up from 13.8 tesla in the nominal $40 \mathrm{~mm}$ aperture mode. This is accomplished by changing/modifying some of the internal support modules inside the iron yoke and using two power supplies. A similar modification in internal structure allows addition and/or replacements of coils made with different size conductor.

A large bend radius in the magnet ends allows this design to accommodate coils made with High Temperature Superconductors (HTS). The advantage of HTS in very high field magnets is the small drop in the critical current density with increasing field. The current density in HTS is not large enough yet to create a high field in a practical design but is large enough to carry out magnet R\&D. An HTS insert coil in a hybrid magnet where the majority of the field comes from conventional low temperature superconductor (e.g. $\mathrm{Nb}_{3} \mathrm{Sn}$ ) will be a first important step towards the future HTSbased accelerator magnet research. This will address the relevant magnetic and mechanical design issues since here the HTS will be in an environment similar to that in a complete HTS magnet.

An overall inodular approach also facilitates a systematic study of magnetic and mechanical design. In the past many such changes required building a new magnet which takes a much longer time and a new magnet does not always reproduce a similar situation. In addition, the horizontal and vertical support, which differ significantly in their design philosophies, can be studied separately here. The same modules can also be used to carry out the high field magnet R\&D for the proposed muon collider [10].

\section{CONCLUSIONS}

A preliminary design of a high field 2-in-1 dipole based on a common coil design has been presented. The construction of this magnet follows the completion of the moderate field magnet now in preparation for test at LBNL. Given a relatively long lead time available for the next large hadron collider, the design philosophy and approach takes advantage of carrying out a systematic and innovative magnet R\&D program to produce lower cost magnets for lower cost hadron colliders.

\section{REFERENCES}

[1] R. Gupta., "A Common Coil Design for High Field 2-in-1 Accelerator Magnets," 1997 Particle Accelerator Conference, Vancouver, Canada, May 1997.

[2] K. Chow, et al., "Design and Fabrication of Racetrack Coil Accelerator Magnets," Sixth European Particle Accelerator Conference, Stockholm, Sweden, June 1998.

[3] K. Chow, et al, "Fabrication and Test Results of a Prototype, $\mathrm{Nb}_{3} \mathrm{Sn}$ Superconducting Racetrack Magnet," Applied Superconductivity Conference, Palm Desert, CA, September 1998.

[4] G. Danby, et al., "Proceedings of $12^{\text {th }}$ International Conference on High Energy," Fermilab, August 11-16, 1983.

[5] S. Caspi et al., "Development of High Field Dipole Magnets for Future Accelerators," Fifteenth International Conference on Magnet Technology, Beijing, China, October 1997.

[6] S. Russenschuck., "ROXIE - A computer Code for the Integrated Design of Accelerator Magnets," Sixth European Particle Accelerator Conference, Stockholm, Sweden, June 1998.

[7] Preis, K. et al., "Numerical Analysis of 3d Magnetostatic Fields," IEEE Transactions on Magnetics, 27, 3798 - 3803, 1991.

[8] K. P. Chow and G. A. Millos, "Measurements of Modulus of Elasticity and Thermal Contraction of Epoxy Impregnated Niobium-Tin and Niobium-Titanium Composites," Applied Superconductivity Conference, Palm Desert, CA, September 1998.

[9] T. Elliot et al., "16 Tesla Nb3Sn Dipole Development at Texas A\&M University," IEEE Transactions on Applied Superconductivity, Vol. 7, \#2, pp. 555-557, June 1997.

[10] R. Gupta, et al., "Nb 3 Sn Magnets for a Muon Collider," Sixth European Particle Accelerator Conference, Stockholm, Sweden, June 1998. 


\section{LEGAL NOTICE}

This report was prepared as an account of work sponsored by the United States Government. Neither the United States nor the Department of Energy, nor any of their employees, nor any of their contractors, subcontractors, or their employees, makes any warranty, express or implied, or assumes any legal liability or responsibility for the accuracy, completeness or usefulness of any information, apparatus, product or process disclosed, or represents that its use would not infringe privately owned rights. 